\title{
Fast Methods for Simulation of Biomolecule Electrostatics
}

\author{
Michael D. Altman, Shihhsien S. Kuo, Jaydeep P. Bardhan, Jacob K. White \& Bruce Tidor
}

Artificial Intelligence Laboratory

Massachusetts Institute of Technology

Cambridge, Massachusetts 02139

http://www.ai.mit.edu

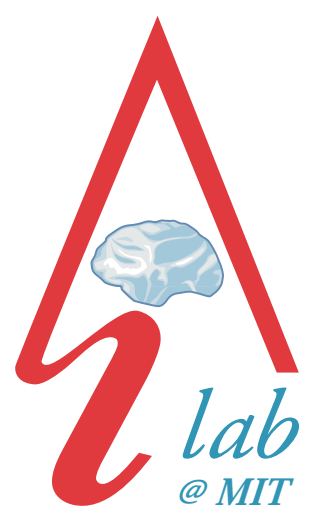

The Problem: Biomolecular structure and interactions in an aqueous environment are determined by a complicated interplay between physical and chemical forces including solvation, electrostatics, van der Waals forces, the hydrophobic effect, and covalent bonding. Electrostatic forces have received a great deal of study due to their longrange nature and the tradeoff between desolvation and interaction effects [1]. In addition, electrostatic interactions play a significant role within a biomolecule as well as between biomolecules, making the balance between the two vital to the understanding of macromolecular systems. Specifically, the goal of this work is to accurately and quickly compute the strength of electrostatic interactions for a biomolecule in an electrolyte solution, as well as the potential field that the molecule generates in all space.

Motivation: There are two valuable uses for these computations. First, it provides a full picture of the electrostatic energetics of a biomolecular system, improving our understanding of how electrostatics contribute to stability, function, and molecular interactions [3]. Second, these simulations serve as a tool for molecular design, since electrostatic complementarity is an important feature of interacting molecules. Through examination of the electrostatics and potential field generated by a protein molecule, for example, it may be possible to suggest improvements to other proteins or drug molecules that interact with it, or perhaps even design new interacting molecules de novo [2].

Previous Work: One popular approach to simulating biomolecule electrostatics in aqueous solution with nonzero ionic strength consists of a mixed discrete-continuum method based on combining a continuum description of the molecule and solvent with a discrete description of the atomic charges [5]. Solutions to the mixed discretecontinuum model are mostly computed numerically, using schemes based on finite-difference discretizations of the model's underlying partial differential equation, the Poisson-Boltzmann equation, generally implemented in its linearized form [1]. Although finite-difference methods have proven to be effective, there are several characteristics of the biomolecule application which are problematic for such methods. Inaccuracies can be generated when projecting the discrete charges, to finite-difference grids. The problem is particularly troublesome when attempting to compute reaction forces at those point charge locations. In addition, the large jump in dielectric constant across the irregularly-shaped protein-solvent boundary must be treated carefully. Finally, the solvent region is unbounded, at least formally, and must be somehow truncated before applying a finite-difference method. Modifications of the basic finite-difference method have been developed to resolve many of these difficulties [1], though often at considerable computational cost.

Approach: In this work we demonstrate that a more efficient procedure can be developed by combining a carefully chosen integral formulation of the mixed discrete-continuum linearized Poisson-Boltzmann model with one of the recently developed fast integral equation solvers [4]. Numerical methods based on solving an integral formulation can treat point charges, irregularly shaped regions with large jumps in parameters, unbounded domains, and the reaction force computation much more naturally than finite-difference methods. Even though integral formulations have many advantages for this application, they are not often used; the available numerical techniques for solving integral equations were too computationally expensive to use on complicated problems, but recently developed fast algorithms have changed that situation considerably. The precorrected-FFT (pFFT) algorithm [4] is an accelerated implementation of the boundary element method (BEM) that is capable of accurately solving the integral equations involved in biomolecular electrostatics. The algorithm can be summarized in four steps where a given set of panels from a discretized surface is superimposed on a uniform grid. First, panel charges are projected onto their associated grid points, in what is called the projection step. Second, given the distribution of grid charges, the grid potential can be calculated using a convolution of the Green's function (the kernel) and the grid charges; this convolution is efficiently computed using the fast Fourier transform (FFT). Third, grid potentials are 
interpolated back onto the panels, a step known as interpolation. In the fourth step, called precorrection, nearby interactions are computed directly, with a correction factor that removes the contributions from the grid. All four steps-projection, interpolation, FFT convolution, and precorrection-possess sparse representations, and so the algorithm is very efficient in both speed (roughly $\Theta(n \log n)$ ) and memory (roughly $\Theta(n)$ ), where $n$ is the number of panels. This is a tremendous improvement over traditional methods for discretizing the integral equations, which generate dense matrices and therefore require $n^{2}$ memory and $n^{2}$ operations for matrix-vector multiplication.

Impact: The integral formulation of the linearized Poisson-Boltzmann equation solved through the precorrectedFFT algorithm has been implemented and applied to several systems. The first case is a hypothetical spherical molecule with analytical results for the potential at its center. The integral equation formulation is well conditioned for this system and converges rapidly to a value within $0.01 \%$ of the analytical solution. The next case is the water molecule, which cannot be solved analytically. The pFFT solver yielded potentials at atom centers within $0.1 \%$ of those calculated with an equivalent finite-difference method. The next application of the pFFT solver was to a highly charged small organic molecule with 26 atoms, the transition state analog (TSA) of the protein enzyme chorismate mutase. Figure 1 shows the relationship between the surface discretization level and the solvation free energy calculated as well as the potential distribution on the molecular surface. An E. coli chorismate mutase (ECM) protein macromolecule with 3210 atoms was also simulated with the pFFT solver. Results are within $1 \%$ of finite-difference methods. The results from these simulations are promising and show good agreement with finitedifference solutions to the same equations. The pFFT solver is up to 30 times faster than finite-difference solvers for the small systems, and about the same speed for large protein systems. This is likely due to a lack of optimization in the implementation.
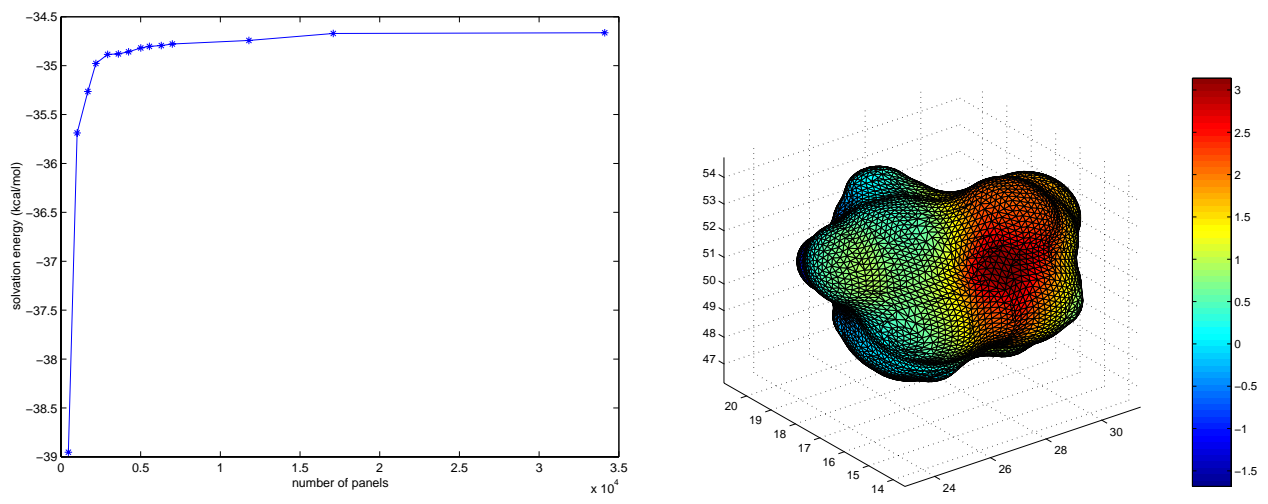

Figure 1: Convergence of solvation energy for the TSA molecule with increasing surface discretization (left) and surface potential on the boundary of the TSA molecule (right).

Future Work: These preliminary results are encouraging and indicate a potential application of this formulation. More rigorous test cases are being designed and studied, and further optimization of the pFFT implementation is under investigation.

Research Support: This work is supported by the Singapore-MIT Alliance, the National Science Foundation, the Defense Advanced Research Projects Agency and the National Institutes of Health.

\section{References:}

[1] M. K. Gilson, K. A. Sharp, and B. H. Honig. Calculating the electrostatic potential of molecules in solution: Method and error assessment. Journal of Computational Chemistry, 9:327-335, 1987.

[2] E. Kangas and B. Tidor. Electrostatic specificity in molecular ligand design. Journal of Chemical Physics, 112:91209131, 2000.

[3] L. P. Lee and B. Tidor. Barstar is electrostatically optimized for tight-binding to barnase. Nature Structural Biology, 8:73-76, 2001.

[4] J. R. Phillips and J. K. White. A precorrected-FFT method for electrostatic analysis of complicated 3-D structures. IEEE Transactions on Computer-Aided Design of Integrated Circuits and Systems, 16:1059-1072, 1997. 
[5] C. Tanford and J. G. Kirkwood. Theory of protein titration curves I. general equations for impenetrable spheres. Journal of the American Chemical Society, 59:5333-5339, 1957. 